\title{
ON SOME PROPERTIES OF SOLUTIONS OF POLYHARMONIC EQUATION IN POLYHEDRAL ANGLES
}

\author{
ILIA TAVKHELIDZE
}

\begin{abstract}
For a higher order differential equation with the polyharmonic operator, the Dirichlet and Riquier boundary value problems are studied in some polyhedral angles. Uniqueness theorems for solutions with a bounded "energy integral" of the corresponding BVPs are proved. Recurrent formulas are constructed for representation of fundamental solutions and Green's functions. The asymptotic behavior of solutions at infinity is studied.
\end{abstract}

2000 Mathematics Subject Classification: 35J40, 35B30, 35B40.

Key words and phrases: Biharmonic equation, polyharmonic equation, Dirichlet problem, Riquier problem, fundamental solution, Green's function.

\section{INTRODUCTION}

The Dirichlet problem in a bounded domain for a polyharmonic equation was studied by S. L. Sobolev [17]-[19]. Dirichlet, Neumann, Riquier and some other BVPs were investigated by I.Vekua [23]-[24] in bounded and unbounded domains for harmonic, biharmonic and metaharmonic functions. At various times and by different methods, many authors studied analogous problems in more general cases (see, e.g., [1]-[15], [20]-[21]. In the present paper, Dirichlet and Riquier problems are studied in some unbounded domains for the higher order elliptic differential equation

$$
\Delta^{m} u(x)=f(x) \quad\left(f \in C_{0}^{\infty}\right)
$$

with the polyharmonic operator on the left-hand side (the first short report about one special case can be found in [20]). In particular, the relation between the asymptotic behavior of solutions of the corresponding BVPs and the righthand side of equations is investigated.

Throughout the paper the following notation is used:

$\mathbb{N}$ is the set of natural numbers and $n$ is a space dimension;

$\Delta^{m}$ is the polyharmonic operator, where $\Delta$ is the Laplace operator;

$m \in \mathbb{N}$ is the order of a polyharmonic operator;

the Greek letters $\alpha, \beta, \ldots$ are multiindexes (e.g., $\alpha \equiv\left(\alpha_{1}, \alpha_{2}, \ldots, \alpha_{n}\right)$ where every $\left.\alpha_{i} \in \mathbb{N} \bigcup\{0\}\right)$;

$D_{i} u(x) \equiv u_{, i}(x) \equiv \frac{\partial u(x)}{\partial x_{i}}, D_{i k} u(x) \equiv u_{, i k}(x) \equiv \frac{\partial^{2} u(x)}{\partial x_{i} \partial x_{k}}$ for every $i, k=\overline{1, n}$;

$D^{\alpha} \equiv D_{1}^{\alpha_{1}} \ldots D_{n}^{\alpha_{n}}$

$|\alpha| \equiv \alpha_{1}+\cdots+\alpha_{n}$ is the module of a multiindex $\alpha$;

$\alpha ! \equiv \alpha_{1} ! \alpha_{2} ! \cdots \alpha_{n}$ ! is the factorial of a multiindex $\alpha$;

the repeated indexes mean that summation is performed over them; 
$\Re_{l}^{n} \equiv\left\{x \equiv\left(x_{1}, x_{2}, \ldots, x_{n}\right) \in \Re^{n}: x_{i} \geq 0, i=n-l, n-l+1, \ldots, n\right\}, l \in$ $\{0,1, \ldots, n-1\}$, are polyhedral angles;

$\Re_{0}^{n} \equiv \Re_{+}^{n} \equiv\left\{x \equiv\left(x_{1}, x_{2}, \ldots, x_{n}\right): x_{n}>0\right\}$ is the half-space;

$E_{m}(u, v) \equiv \sum_{|\alpha|=m} \frac{m !}{\alpha !} D^{\alpha} v D^{\alpha} u$ for every nonnegative integer $m$ and for all functions $u(x)$ and $v(x)$;

$E_{m}(u) \equiv E_{m}(u, u)$

$|x| \equiv \sqrt{x_{1}^{2}+x_{2}^{2}+\ldots+x_{n}^{2}}$

$Q_{\rho} \equiv \Re_{l}^{n} \cap\left\{x \in \Re_{l}^{n}:|x|<\rho\right\}$; for $l=0, Q_{\rho}$ is a semiball of radius $\rho$ centered at the origin;

$\delta(x)$ is the Dirac function;

$\Lambda_{n \mid m}(x)$ is a fundamental solution of the polyharmonic equation of order $m$, $n$ is a space dimension;

$G_{n \mid m}(x, y)$ is the Green's function for the Dirichlet problem for a polyharmonic equation of order $m, n$ is a space dimension.

Let $\omega$ be an open set in $\Re_{l}^{n}$ and $\gamma$ be a subset of its boundary $\gamma \subset \partial \omega$. If $\omega$ is a bounded domain, denote by $H_{2}(\omega, \gamma)$ the Sobolev space obtained by completion of the set of $m$ times continuously differentiable functions $u$ on $\bar{\omega}$ that equal zero in a neighborhood of $\gamma$ with respect to the norm

$$
\|u\|_{m} \equiv\left[\int_{\omega} \sum_{|\alpha| \leq m}\left(D^{\alpha} u\right)^{2} d x\right]^{\frac{1}{2}} .
$$

But if $\omega$ is an unbounded domain, then the definition of $H_{2}(\omega, \gamma)$ applies with $u(x)=0$ also at the intersection of $\omega$ with some neighborhood of the point at infinity.

Definition 1.1. We say that a function $u(x)$ is a generalized solution of the equation (1.1) in $\Re_{l}^{n}$ with homogeneous Dirichlet boundary conditions if $u \in H_{m}\left(\Re_{l}^{n}\right)$ and if it satisfies the integral identity

$$
(-1)^{m} \int_{Q_{\rho}} E_{m}(u, v) d x=\int_{Q_{\rho}} f(x) v(x) d x
$$

for any $\rho>0$ and any function $v \in H_{m}\left(Q_{\rho}, \partial Q_{\rho}\right)$, where $f \in L_{2}\left(\Re_{l}^{n}\right)$.

In this paper we prove the uniqueness theorems based on the "generalized Hardy's inequality". The formulation and proof of this inequality can be found, e.g., in the paper by V. A. Kondratiev and O. A. Oleunik [10].

Lemma 1.1 (Generalized Hardy's inequality). Let the numbers $j, n$ and $p \in$ $(0, \infty)$ be such that $j+n-p \neq 0$. If for a sufficiently smooth function $g(x)$ in a cone $V \subset \Re^{n}$ with vertex at the origin, the following condition

$$
\int_{V}\left|x^{j}\right||\nabla g(x)|^{p} d x<\infty
$$


is fulfilled, where $\nabla \equiv\left(\frac{\partial}{\partial x_{1}}, \ldots, \frac{\partial}{\partial x_{n}}\right)$ is the gradient vector, then there exists some constant $M$ such that

$$
\int_{V}\left|x^{j-p}\right||g(x)-M|^{p} d x<K \int_{V}\left|x^{j}\right||\nabla g(x)|^{p} d x,
$$

where the constant $K$ is independent of the function $g(x)$. If, in addition, $g(0)=$ 0 , then the constant $M=0$.

\section{On the Dirichlet and Riquier Problems for Biharmonic EQUATION IN THE HALF-SPACE}

We consider, in the half-space $\Re_{+}^{n}$ Sophie Germain's equation (the particular case of equation (1.1) when $m=2$ )

$$
\Delta^{2} u(x)=f(x)\left(f \in C_{0}^{\infty}\left(\Re_{+}^{n}\right)\right)
$$

with the homogeneous boundary conditions

$$
\left.u\right|_{x_{n}=0}=0,\left.\quad \frac{\partial u}{\partial x_{n}}\right|_{x_{n}=0}=0 \quad \text { (Dirichlet problem) }
$$

or

$$
\left.u\right|_{x_{n}=0}=0,\left.\quad \frac{\partial^{2} u}{\partial x_{n}^{2}}\right|_{x_{n}=0}=0 \quad \text { (Riquier problem). }
$$

We assume that all solutions of both problems have a finite "energy integral" (i.e., for all solutions of both BVPs the condition

$$
\int_{\Re_{+}^{n}} E_{2}(u) d x<\infty
$$

holds and sometimes an additional condition

$$
\int_{\Re_{+}^{n}} E_{1}(u) d x<\infty
$$

is assumed to be fulfilled).

Remark 2.1. It is easy to check that every classical (smooth) solution of the problem (2.1)-(2.2) is also a generalized solution. It is likewise easy to see that the classical solution of the Riquier problem (2.1)-(2.3) satisfies the integral identity (1.2) for any semiball $Q_{\rho}$ and for any function $v \in H_{2}\left(Q_{\rho}, \partial Q_{\rho}\right)$.

We begin our discussion by proving the uniqueness theorems of solutions of the corresponding BVPs.

Theorem 2.1 (Uniqueness of a Generalized solution of the Dirichlet BVP). Let the function $u(x)$ be a generalized solution of the homogeneous equation (2.1) (i.e., $f(x) \equiv 0$ ) with the boundary conditions (2.2). Then $u(x) \equiv 0$ in $\Re_{+}^{n}$ for any $n>2$. 
Proof. Let us consider an auxiliary function

$$
\Theta(s) \equiv \begin{cases}1, & 0<s<1, \\ \theta(s), & 1<s<2, \quad 0<\theta(s)<1, \quad \Theta \in C_{0}^{\infty}(0, \infty), \\ 0, & s>2\end{cases}
$$

such that

$$
\left|\Theta^{\prime}(s)\right|^{2} \leq K_{0} \Theta(s), \quad K_{0}=\text { const }>0 .
$$

For any $R>0$ let

$$
\Theta_{R}(x) \equiv \Theta\left(\frac{|x|}{R}\right)
$$

Note that for any numbers $i, k=\overline{1, n}$ the following relations hold:

$$
\begin{gathered}
\Theta_{R, i}(x) \equiv \frac{\partial \Theta_{R}(x)}{\partial x_{i}}=\Theta^{\prime}(|x|) \cdot \frac{x_{i}}{R|x|} \\
\Theta_{R, i k}(x)=\Theta^{\prime \prime}(|x|) \cdot \frac{x_{i} \cdot x_{k}}{R^{2}|x|^{2}}+\Theta^{\prime}(|x|) \cdot \frac{|x|^{2} \cdot \epsilon_{i k}-x_{i} \cdot x_{k}}{R|x|^{3}},
\end{gathered}
$$

where $\epsilon_{i k}$ is the Kronecker symbol.

Consider now $v(x) \equiv u(x) \cdot \Theta_{R}(x)$. Note that, by the boundary conditions (2.2), due to (2.8) we have

$$
v \in H_{2}\left(Q_{2 R}, \partial Q_{2 R}\right)
$$

Substituting this function into the integral identity (1.2), we obtain

$$
\int_{Q_{2 R}} u_{, i k} u_{, i k} \Theta_{R}(x) d x+2 \int_{Q_{2 R} \backslash Q_{R}} u_{, i k} u_{, i} \Theta_{R, k}(x) d x+\int_{Q_{2 R} \backslash Q_{R}} u_{, i k} u \Theta_{R, i k}(x) d x=0 .
$$

According to our notation and formulas (2.7)-(2.10) this relation can be rewritten as

$$
\int_{Q_{2 R}} E_{2}(u) \cdot \Theta_{R}(x) d x=\left|2 \int_{Q_{2 R} \backslash Q_{R}} u_{, i k} u_{, i} \Theta^{\prime}(|x|) \frac{x_{k}}{R|x|} d x+\int_{Q_{2 R} \backslash Q_{R}} u_{, i k} u \Theta_{R, i k}(x) d x\right| .
$$

Using a simple inequality

$$
a b \leq \frac{\varepsilon \cdot a^{2}}{2}+\frac{b^{2}}{2 \varepsilon}
$$

which holds for every $\varepsilon=$ const $>0$ and for any numbers $a, b \in \Re^{1}$, and taking into account (2.7)-(2.10) we obtain

$$
\begin{aligned}
\int_{Q_{2 R}} E_{2}(u) \cdot \Theta_{R}(x) d x \leq & \left(\varepsilon+\varepsilon_{1}\right) \int_{Q_{2 R} \backslash Q_{R}} E_{2}(u) \cdot \Theta_{R} d x \\
& +\frac{K_{1}}{\varepsilon} \int_{Q_{2 R} \backslash Q_{R}} \frac{u_{, i} u_{, i}}{|x|^{2}} d x+\frac{K_{2}}{\varepsilon_{1}} \int_{Q_{2 R} \backslash Q_{R}} \frac{u^{2}}{|x|^{4}} d x,
\end{aligned}
$$


here the constants $K_{1}$ and $K_{2}$ are independent of the function $u(x)$ and of radius $R$, while $\varepsilon$ and $\varepsilon_{1}$ are arbitrary constants from relation (2.11). Applying Hardy's inequality (1.4) to the third term on the right-hand side of (2.12) when $p=2$ and $j=-2$, we have

$$
\int_{Q_{2 R}} E_{2}(u) \cdot \Theta_{R}(x) d x \leq\left(\varepsilon+\varepsilon_{1}\right) \int_{Q_{2 R} \backslash Q_{R}} E_{2}(u) \cdot \Theta_{R} d x+\left[\frac{K_{1}}{\varepsilon}+\frac{K_{2}^{*}}{\varepsilon_{1}}\right] \int_{Q_{2 R} \backslash Q_{R}} \frac{u_{, i} u_{, i}}{|x|^{2}} d x .
$$

Applying Hardy's inequality (1.4) to the second term on the right-hand side implies the estimate

$$
\int_{Q_{2 R}} E_{2}(u) \cdot \Theta_{R}(x) d x \leq \widetilde{K} \int_{Q_{2 R} \backslash Q_{R}} E_{2}(u) d x
$$

where the constant $\widetilde{K}$ does not depend on the radius $R$ and on the function $u(x)$. Consequently, for any positive number $P>0$ and any radius $R>P$, due to the last relation we obtain

$$
\int_{Q_{P}} E_{2}(u) d x \leq \widetilde{K} \int_{Q_{2 R} \backslash Q_{R}} E_{2}(u) d x .
$$

Since $u$ is a generalized solution, condition (2.4) is automatically fulfilled and the right-hand side of (2.13) tends to zero as $R \longrightarrow \infty$. Note that the left-hand side of $(2.13)$ is independent of the radius $R$. Thus we have obtained

$$
\int_{Q_{P}} E_{2}(u) d x \equiv \int_{Q_{P}} u_{, i k} u_{, i k} d x \equiv 0
$$

for any positive number $P$.

Remark 2.2. When $n=2$ (half-plane) one can easily check that, due to (2.4), condition (1.3) holds, and $j+n-p=0(j=0$ and $p=2)$. Thus the generalized Hardy's inequality is not true. Hence in this paper the uniqueness theorem is proved only for the case where a space dimension is $n \geq 3$.

A similar reasoning leads to

Theorem 2.2 (Uniqueness of a solution of the Riquier BVP). Let the function $u(x)$ be a classical solution of the homogeneous equation (2.1) (i.e., $f(x) \equiv$ $0)$ with the boundary conditions (2.3) in the half-space $\Re_{+}^{n}$ and $n>2$. If the additional condition (2.4) is fulfilled, then $u(x)=\widehat{K} x_{n}$ in $\Re_{+}^{n}$ with an arbitrary constant $\widehat{K}$. But if, in addition, condition (2.5) is fulfilled too, then the solution is unique and $u(x) \equiv 0$.

Proof. Consider now a function $v(x) \equiv\left(u(x)-\widehat{K} x_{n}\right) \cdot \Theta_{R}(x)$, where $\Theta_{R}(x)$ is defined in (2.8). Substituting this function into the integral identity (1.2), as in 
the previous proof, we obtain

$$
\begin{aligned}
& \int_{Q_{2 R}} E_{2}(u) \cdot \Theta_{R}(x) d x \leq\left(\varepsilon+\varepsilon_{1}\right) \int_{Q_{2 R} \backslash Q_{R}} E_{2}(u) \cdot \Theta_{R} d x \\
& \quad+\frac{K_{1}}{\varepsilon} \int_{Q_{2 R} \backslash Q_{R}} \frac{\left(u-\widehat{K} x_{n}\right)_{, i}\left(u-\widehat{K} x_{n}\right)_{, i}}{|x|^{2}} d x+\frac{K_{2}}{\varepsilon_{1}} \int_{Q_{2 R} \backslash Q_{R}} \frac{\left(u-\widehat{K} x_{n}\right)^{2}}{|x|^{4}} d x .
\end{aligned}
$$

Hence every second order derivative of the solution of the BVP (2.1)-(2.3) equals zero. Therefore, from the boundary condition (2.3) it follows that $u(x)=\widehat{K} x_{n}$. It is easy to verify that if the additional condition (2.5) is fulfilled, then $u(x) \equiv$ 0 .

Remark 2.3. One can easily check that these theorems are true for any unbounded conical domain with vertex at the origin.

Now let as study the structure of solutions of the corresponding BVPs and the relationship between the function on the right-hand side of equation (2.1) and the character of the asymptotic behavior of these solutions.

Remark 2.4. It is well-known that in the half-space the Polyharmonic function $u(x)$ admits two different representations:

- I. Almazi's representation (see, e.g., [23] or [1])

$$
u(x)=\sum_{j=1}^{m}|x|^{2(m-j)} v_{j}(x),
$$

- II. The representation (see, e.g., [14] or [22])

$$
u(x)=\sum_{j=1}^{m} x_{n}^{(m-j)} w_{j}(x),
$$

where $v_{j}(x)$ and $w_{j}(x)$ are harmonic functions for any $j=\overline{1, m}$.

We have generalized one proposition from [23] proved by I. Vekua in the two-dimensional case.

Proposition 2.1. If $\Lambda_{n \mid 1}(x)$ is a fundamental solution of Laplace equation, then

$$
\Lambda_{n \mid 2}(x) \equiv \frac{|x|^{2}}{2(4-n)} \Lambda_{n \mid 1}(x)
$$

is a fundamental solution of the biharmonic equation except the case with $n=4$.

Proof. By the theory of distributions (of generalized functions [16]),

$$
\begin{aligned}
& \Delta \Delta\left(|x|^{2} \Lambda_{n \mid 1}(x)\right)=\Delta\left[2 n \Lambda_{n \mid 1}(x)+|x|^{2} \Delta \Lambda_{n \mid 1}(x)+4 x_{k} \Lambda_{n \mid 1, k}(x)\right] \\
& \quad=4 n \Delta \Lambda_{n \mid 1}(x)+|x|^{2} \Delta \Delta \Lambda_{n \mid 1}(x)+8 x_{k} \Delta \Lambda_{n \mid 1, k}(x)+8 x_{k, j} \Lambda_{n \mid 1, k j}(x) .
\end{aligned}
$$


Recall that summation is assumed to be taken from 1 to $n$ over the repeated indexes. Therefore for every "basic" function $\left(\varphi \in C_{0}^{\infty}\left(\Re^{n}\right)\right)$, in view of the formulas (3.13) and (3.14) below, the following relation holds

$$
\left\langle\Delta \Delta\left(|x|^{2} \Lambda_{n \mid 1}(x)\right) \mid \varphi(x)\right\rangle=2(4-n)\langle\delta(x) \mid \varphi(x)\rangle,
$$

which proves the proposition.

By our notation, $G_{n \mid 1}(x, y)$ is the classical Green's function for the Dirichlet problem for the Laplace equation in the half-space, i.e.,

$$
G_{n \mid 1}(x, y)=\frac{1}{\sigma_{n}(n-2)}\left[\frac{1}{|x-y|^{n-2}}-\frac{1}{|x-\bar{y}|^{n-2}}\right],
$$

where $\sigma_{n}$ is the surface area of the $n$-dimensional ball of radius 1 , and $\bar{y} \equiv$ $\left(y_{1}, \ldots, y_{n-1},-y_{n}\right)$ for $y=\left(y_{1}, \ldots, y_{n}\right)$.

Theorem 2.3. The generalized unique solution of the BVP (2.1), (2.2) in the half-space $\Re_{+}^{n}$ has the form

$$
u(x)=\int_{\Re_{+}^{n}} G_{n \mid 2}(x, y) f(y) d y
$$

with

$$
G_{n \mid 2}(x, y)= \begin{cases}\frac{1}{2(4-n)}\left[|x-y|^{2} G_{n \mid 1}(x, y)+\frac{2 x_{n} y_{n}}{\sigma_{n}|x-\bar{y}|^{n-2}}\right], & n \neq 4, \\ c_{4}\left[\ln \frac{1}{|x-y|}-\ln \frac{1}{|x-\bar{y}|}\right]-\frac{2 c_{4} x_{4} y_{4}}{|x-\bar{y}|^{2}}, & n=4,\end{cases}
$$

where $c_{4}$ is a constant.

Proof. Firstly, note that the Green's function (2.19) is a fundamental solution of the biharmonic equation (2.1). Due to the structure of this function, for any $n$ the second term on the right-hand side of (2.19) is the first term of the representation (2.15), and hence this term is a biharmonic function; for $n \neq 4$ the first term on the right-hand side of (2.19) is the first term of Almanzi's representation (2.15) and so this term is a fundamental solution, i.e.,

$$
\Delta^{2} G_{n \mid 2}(x, y)=\delta(x)
$$

If $n=4$, then, as is easy to check by simple calculations, the second term on the right-hand side of (2.19) is a fundamental solution, i.e., identity (2.20) holds.

Secondly, the Green's function (2.19) satisfies the boundary conditions (2.2). In that case, the boundary of the domain is the hyperspace $x \in \Re_{+}^{n}: x_{n}=0$ and hence in both cases the second term of (2.19) is zero. Note that

$$
|x-\bar{y}|=|x-y| \quad \text { when } \quad x_{n}=0 .
$$


and therefore the first Dirichlet condition (2.2) holds. It is easy to check that if $n \neq 4$, then

$$
\begin{aligned}
\frac{\partial G_{n \mid 2}(x, y)}{\partial x_{n}}= & \frac{\left(x_{n}-y_{n}\right)}{4-n} G_{n \mid 1}(x, y)+|x-y|^{2} \frac{\partial G_{n \mid 1}(x, y)}{\partial x_{n}} \\
& +\frac{2 y_{n}}{\sigma_{n}|x-\bar{y}|^{(n-2)}}+\frac{2 x_{n} y_{n}}{\sigma_{n}} \frac{\partial|x-\bar{y}|^{(2-n)}}{\partial x_{n}}
\end{aligned}
$$

If $x_{n}=0$, the first term on the right-hand side of (2.22) equals zero, since $G_{n \mid 1}(x, y)$ is the Green's function of the Dirichlet BVP for the Laplace equation. It is evident that the fourth term on the right-hand side of (2.22) also equals zero. Due to (2.17) simple calculations give

$$
|x-y|^{2} \frac{\partial G_{n \mid 1}(x, y)}{\partial x_{n}}=\frac{|x-y|^{2}}{(n-2)}\left[\frac{-(n-2)\left(x_{n}-y_{n}\right)}{|x-y|^{n}}-\frac{-(n-2)\left(x_{n}+y_{n}\right)}{|x-\bar{y}|^{n}}\right] \text {. }
$$

Hence from (2.21) we have

$$
|x-y|^{2} \frac{\partial G_{n \mid 1}(x, y)}{\partial x_{n}}=-\frac{2 y_{n}}{\sigma_{n}|x-\bar{y}|^{(n-2)}}
$$

and thus the second and the third term on the right-hand side of (2.22) vanish. Therefore the second Dirichlet condition (2.2) is fulfilled. By simple calculations it is easy to check that for $n=4$ the function $G_{4 \mid 2}(x, y)$ also satisfies the second boundary condition (2.2).

Thirdly, it is easy to see that for any $i, k=\overline{1, n}$ the partial derivative $\frac{\partial^{2} u}{\partial x_{i} \partial x_{k}}$ of the solution (2.18) has the order of decay $|x|^{1-n}$ at infinity. Therefore for the solution (2.18) inequality (2.4) is fulfilled when $n>2$. By uniqueness theorem 2.1 the solution (2.18) of the BVP $(2.1),(2.2)$ is unique.

A solution of the Riquier BVP is constructed similarly.

Theorem 2.4. The function

$$
u(x)=\int_{\Re_{+}^{n}} G_{n \mid 2}^{*}(x, y) f(y) d y
$$

with

$$
\begin{aligned}
& G_{n \mid 2}^{*}(x, y) \\
& = \begin{cases}\frac{|x-y|^{2}}{2(4-n)} G_{n \mid 1}(x, y)+\frac{2 x_{n} y_{n}}{\sigma_{n}(4-n)(2-n)|x-\bar{y}|^{n-2}}, & n \neq 2 \text { or } 4, \\
\frac{|x-y|^{2}}{4} G_{2 \mid 1}(x, y)-\frac{4 x_{2} y_{2}}{\pi} \ln \left(\frac{1}{|x-y|}\right), & n=2, \\
c_{4}\left[\ln \frac{1}{|x-y|}-\ln \frac{1}{|x-\bar{y}|}\right], & n=4,\end{cases}
\end{aligned}
$$

is a classical unique solution of the $B V P(2.1)$, (2.3) if $n>4$ and conditions (2.4) and (2.5) hold in the half-space $\Re_{+}^{n}$; here $c_{4}$ is a constant defined in (2.19). 
In the cases $n=3,4$ this solution is unique to within an additional term $c x_{n}$, where $c$ is an arbitrary constant.

Proof. The proof of this theorem proceeds similarly to that of the previous one. But note that in this case for any $i=\overline{1, n}$, the partial derivative $\frac{\partial u}{\partial x_{i}}$ of the solution (2.18) has the order of decay $|x|^{2-n}$ at infinity. Therefore condition (2.5) is fulfilled when $n \geq 5$ and it is only in this case that by Theorem 2.1 the solution (2.23) is unique. Also, by the second boundary condition in (2.3), we do not have a unique solution for $n=3,4$.

Remark 2.5. Neither of these theorems includes the case $n=2$. For the solutions (2.18) and (2.23) condition (2.4) does not take place and therefore these solutions are not unique solutions of the corresponding BVPs.

Now let us consider the asymptotic behavior of these solutions.

Theorem 2.5. If $u(x)(2.18)$ is a solution of the BVP (2.1), (2.2), then

$$
u(x)=M_{n}\left[\int_{\Re_{+}^{n}} y_{n}^{2} f(y) d y\right] \cdot \frac{x_{n}^{2}}{|x|^{n}}+O\left(|x|^{1-n}\right),
$$

where $M_{n}=\frac{2}{\sigma_{n}(4-n)}$ if $n \neq 4$ and $M_{4}=2 c_{4}$ if $n=4$, and $c_{4}$ is defined in (2.19).

Proof. Note that for the solution (2.19) the coefficients of the first terms of Taylor's series in the neighborhood of $y=0$ are

$$
G_{n \mid 2}(x, 0)=\left.\frac{\partial G_{n \mid 2}(x, y)}{\partial y_{k}}\right|_{y=0}=\left.\frac{\partial^{2} G_{n \mid 2}(x, y)}{\partial y_{k} \partial y_{j}}\right|_{y=0}=0
$$

for every $k=\overline{1, n}$ and $j=\overline{1, n-1}$. But

$$
\left.\frac{\partial^{2} G_{n \mid 2}(x, y)}{\partial y_{n}^{2}}\right|_{y=0}=M_{n} \cdot \frac{x_{n}^{2}}{|x|^{n}}
$$

\section{Corollary 2.1.}

- I. Half-plane. If $n=2$, then the BVP (2.1), (2.2) has a solution of the form (2.18), but this function does not always tend to zero as $x \longrightarrow \infty$ :

$$
\begin{aligned}
& u\left(x_{1}, x_{2}\right) \longrightarrow 0 \text { when } x_{1} \longrightarrow \infty \text { and } x_{2}=\text { const }, \\
& u\left(x_{1}, x_{2}\right) \longrightarrow \text { const, when } x_{2} \longrightarrow \infty \text { and } x_{1}=\text { const. }
\end{aligned}
$$

- II. Half-space. If $n>2$, then the BVP (2.1), (2.2) has a solution of the form (2.18), this function always tends to zero as $x \longrightarrow \infty$, but the order of its decay is greater than that of fundamental solutions at least by 1 :

$$
\begin{aligned}
& u\left(x_{1}, \ldots, x_{n}\right)=O\left(|x|^{-n}\right) \quad \text { as } \quad|x| \longrightarrow \infty \quad \text { and } \quad x_{n}=\text { const }, \\
& u\left(x_{1}, \ldots, x_{n}\right)=O\left(|x|^{2-n}\right) \quad \text { as } \quad x_{n} \longrightarrow \infty .
\end{aligned}
$$


Theorem 2.6. If $u(x)$ in (2.23) is a solution of the BVP (2.1), (2.3), then

$$
u(x)=M_{n}^{*}\left[\int_{\Re_{+}^{n}} y_{n} f(y) d y\right] \cdot \frac{x_{n}}{|x|^{n}}+O\left(|x|^{2-n}\right),
$$

where $M_{n}^{*}=\frac{1}{\sigma_{n}(2-n)}$ if $n \neq 2,4$ and $M_{4}^{*}=2 c_{4}$ if $n=4$, and $c_{4}$ is defined in (2.19).

Proof. Note that for the solution (2.23) the coefficients of the first terms of Taylor's series in the neighborhood of $y=0$ are

$$
G_{n \mid 2}^{*}(x, 0)=\left.\frac{\partial G_{n \mid 2}^{*}(x, y)}{\partial y_{k}}\right|_{y=0}=0
$$

for every $k=\overline{1, n-1}$. But

$$
\left.\frac{\partial G_{n \mid 2}^{*}(x, y)}{\partial y_{n}}\right|_{y=0}=M_{n}^{*} \cdot \frac{x_{n}}{|x|^{n-2}} .
$$

Corollary 2.2. The structure of the solution (2.23) is asymmetric, but then the behavior of solutions coincides with that of fundamental solutions.

\section{On Some Properties of Solutions of Dirichlet Problems for Polyharmonic Equation in Polyhedral Angles}

In the domain $\Re_{l}^{n}$ the Dirichlet problem

$$
\left.\frac{\partial^{j} u(x)}{\partial \vec{\nu}^{j}}\right|_{\partial \Re_{l}^{n}}=0, \quad j=0, \ldots, m-1,
$$

is considered for equation (1.1), where $\vec{\nu}$ is a unit outer normal to $\partial \Re_{l}^{n}$.

Remark 3.1. It is easy to check that a classical (smooth) solution of problem (1.1)-(3.1) is also a generalized solution.

Recall that due to Definition 1.1 every solution of BVP (3.1) has a finite "energy integral".

$$
\int_{\Re_{l}^{n}} E_{m}(u) d x<\infty .
$$

Theorem 3.1 (Uniqueness of a Generalized solution of the Dirichlet BVP). Let the function $u(x)$ be a generalized solution of the homogeneous equation (1.1) (i.e., $f(x) \equiv 0$ ) with the boundary conditions (3.1). Then $u(x) \equiv 0$ in $\Re_{l}^{n}$.

Proof. It is easy to check that for the function $\Theta_{R}$ defined in (2.8) and for any multiindex $\alpha$ we have

$$
\left|D^{\alpha} \Theta_{R}(x)\right|^{2} \leq \frac{K_{\alpha} \Theta(|x|)}{|x|^{2|\alpha|}}, \quad \text { where } \quad R<|x|<2 R \text {. }
$$


Here the constants $K_{\alpha}$ depend only on order $\alpha$ of a partial differential and $R$ is an arbitrary positive number. Note that $v(x) \equiv u(x) \cdot \Theta_{R}(x) \in H_{m}\left(Q_{2 R}, \partial Q_{2 R}\right)$. Substituting this function into the integral identity (1.2), we obtain

$$
\begin{aligned}
\int_{Q_{2 R}} E_{m}\left(u, u \Theta_{R}\right) d x & =\int_{Q_{2 R}}\left[\sum_{|\alpha|=m} \frac{m !}{\alpha !} D^{\alpha} u D^{\alpha} u\right] \Theta_{R} d x \\
& +\int_{Q_{2 R} \backslash Q_{R}} \sum_{|\alpha|=m} \frac{m !}{\alpha !} D^{\alpha} u\left[\sum_{\beta+\iota=\alpha \iota \neq 0} \frac{(|\beta|+|\iota|) !}{\beta ! \iota !} D^{\beta} u D^{\iota} \Theta_{R}\right] d x=0 .
\end{aligned}
$$

Therefore

$$
\int_{Q_{2 R}} E_{m}(u) \Theta_{R} d x=\left|\int_{Q_{2 R} \backslash Q_{R}} \sum_{|\alpha|=m} \frac{m !}{\alpha !} D^{\alpha} u\left[\sum_{\beta+\iota=\alpha \iota \neq 0} \frac{(|\beta|+|\iota|) !}{\beta ! \iota !} D^{\beta} u D^{\iota} \Theta_{R}\right] d x\right| .
$$

(2.11) and (3.3) imply that

$$
\begin{aligned}
\int_{Q_{2 R}} E_{m}(u) \Theta_{R}(x) d x \leq & \sum_{|\alpha|=m} \frac{m !}{\alpha !} \varepsilon_{\alpha} \int_{Q_{2 R} \backslash Q_{R}} E_{m}(u) \Theta_{R}(x) d x \\
& +\sum_{|\alpha|=m} \frac{m !}{\alpha !} \cdot \frac{K_{\alpha}}{\varepsilon_{\alpha}} \int_{Q_{2 R} \backslash Q_{R}}\left[\sum_{|\beta|=0}^{m-1} E_{|\beta|}(u) \cdot \frac{1}{R^{2(m-|\beta|)}}\right] d x,
\end{aligned}
$$

where the constants $K_{\alpha}$ are defined in (3.3) and $\varepsilon_{\alpha}$ are arbitrary positive numbers from (2.11).

Every domain $\Re_{l}^{n}$ is "conical" with the vertex at the origin. So, we apply repeatedly the Hardy's inequality (1.4) to the second term on right-hand side of the latter inequality until the order of the partial differential achieves the order $m$. Note that the generalized Hardy's inequality holds if $n \geq 2, p=2$ and $j \neq 0$. After an appropriate choice of $\varepsilon_{\alpha}$ we have

$$
\int_{Q_{2 R}} E_{m}(u) \Theta_{R}(x) d x \leq \widetilde{K} \int_{Q_{2 R} \backslash Q_{R}} E_{m}(u) d x .
$$

The constant $\widetilde{K}$ does not depend on the radius $R$ and the function $u(x)$. For an arbitrary real number $P$ there exist a radius $R>P$ and $Q_{P} \subset Q_{R}$. Therefore

$$
\int_{Q_{P}} E_{m}(u) d x \leq \widetilde{K} \int_{Q_{2 R} \backslash Q_{R}} E_{m}(u) d x .
$$

Note that by the condition (3.2), the right-hand side of (3.4) tends to zero as $R \rightarrow 0$. On the other hand, the left-hand side of (3.4) does not depend on $R$. Hence for any positive number $P$ the "energy integral" in the domain $Q_{P}$ equals zero, i.e.,

$$
\int_{Q_{P}} E_{m}(u) d x \equiv \int_{Q_{P}}\left[\sum_{|\alpha|=m} \frac{m !}{\alpha !} D^{\alpha} u D^{\alpha} u\right] d x \equiv 0 .
$$


Therefore partial differentials of any order of solutions equal zero and due to the structure of domains and the boundary conditions (3.1) we deduce that $u(x) \equiv 0$ in $\Re_{l}^{n}$.

To construct the Green's function for the corresponding problems let us consider

Proposition 3.1. If $\Lambda_{n \mid m-1}(x)$ is a fundamental solution of polyharmonic equation

$$
\Delta^{m-1} \Lambda_{n \mid m-1}(x)=\delta(x)
$$

then the function

$$
\Lambda_{n \mid m}(x)=\frac{|x|^{2}}{2(m-1)(2 m-n)} \Lambda_{n \mid m-1}(x)
$$

is a fundamental solution of the polyharmonic equation (1.1) except the case where the order of the equation coincides with the space dimension (i.e., $n=$ $2 m$ ). In this particular case, the fundamental solution is constructed separately.

Proof. For $m=2$ this proposition holds, see (2.16). For any sufficiently smooth functions $V(x)$ and $U(x)$

$$
\Delta(V(x) U(x))=U(x) \Delta V(x)+V(x) \Delta U(x)+2 V_{, k}(x) U_{, k}(x) .
$$

Substituting the function (3.6) multiplied by $c_{n m}^{-1}$, where

$$
c_{n m}=[2(m-1)(2 m-n)]^{-1}, \quad n>1, \quad n \neq 2 m
$$

into equation (1.1) and using induction with respect to the order $m$ of the Laplace operator, we have

$$
\begin{aligned}
\Delta^{m} c_{n m}^{-1} \Lambda_{n \mid m}(x) & \equiv \Delta^{m-1}\left[\Delta|x|^{2} \Lambda_{n \mid m-1}(x)\right] \\
& =\Delta^{m-1}\left[2 n \Lambda_{n \mid m-1}(x)+|x|^{2} \Delta \Lambda_{n \mid m-1}(x)+4 x_{, k} \Lambda_{n \mid m-1, k}\right] \\
& =2 n \delta(x)+\Delta^{m-1}\left[\left|x^{2}\right| \Delta \Lambda_{n \mid m-1}(x)+4 x_{k} \Lambda_{n \mid m-1, k}(x)\right] .
\end{aligned}
$$

Next,

$$
\begin{aligned}
\Delta^{m} c_{n m}^{-1} \Lambda_{n \mid m}(x)=2 n \delta(x) & +\Delta^{m-2}\left[2 n \Delta \Lambda_{n \mid m-1}(x)+|x|^{2} \Delta^{2} \Lambda_{n \mid m-1}(x)\right. \\
& \left.+8 x_{k} \Delta \Lambda_{n \mid m-1, k}(x)+8 \epsilon_{j k} \Lambda_{n \mid m-1, j k}(x)\right],
\end{aligned}
$$

where $\epsilon_{k j}$ is the Kronecker symbol and hence

$$
\begin{aligned}
\Delta^{m} c_{n m}^{-1} \Lambda_{n \mid m}(x)= & 2 \cdot 2 n \delta(x)+\Delta^{m-2}\left(|x|^{2} \Delta^{2} \Lambda_{n \mid m-1}(x)\right. \\
& \left.+2 \cdot 4 x_{k} \Delta \Lambda_{n \mid m-1, k}(x)\right)+8 \delta(x) .
\end{aligned}
$$

In the general case, similarly,

$$
\begin{aligned}
& \Delta^{m} c_{n m}^{-1} \Lambda_{n \mid m}(x)=\iota \cdot 2 n \delta(x) \\
& \quad+\Delta^{m-\iota}\left(|x|^{2} \Delta^{\iota} \Lambda_{n \mid m-1}(x)+\iota \cdot 4 x_{k} \Delta^{\iota-k} \Lambda_{n \mid m-1, k}(x)\right)+8\left[\sum_{j=0}^{\iota-1} j\right] \delta(x)
\end{aligned}
$$


for each number $\iota=\overline{1, m}$ and if $\iota=m$, then

$$
\Delta^{m} c_{n m}^{-1} \Lambda_{n \mid m}(x)=m \cdot 2 n \delta(x)+|x|^{2} \Delta \delta(x)+m \cdot 4 x_{k} \delta_{, k}+8\left[\sum_{j=0}^{m-1} j\right] \delta(x) .
$$

According to the theory of generalized functions, for every "basic" function $\phi \in C_{0}^{\infty}\left(\Re_{l}^{n}\right)$ we have

$$
\left\langle|x|^{2} \cdot \Delta \delta(x) \mid \phi(x)\right\rangle=2 n\langle\delta(x) \mid \phi(x)\rangle
$$

and

$$
\left\langle x_{k} \cdot \frac{\partial \delta(x)}{\partial x_{k}} \mid \phi(x)\right\rangle=-n\langle\delta(x) \mid \phi(x)\rangle .
$$

By (3.9)-(3.10) and (3.12)-(3.13) we rewrite relation (3.11) as

$$
\Delta^{m} c_{n m}^{-1} \Lambda_{n \mid m}(x)=[2 n m+2 n-4 m n+4 m(m-1)] \delta(x) .
$$

By induction with respect to $m$, we deduce that $\Lambda_{n \mid m}(x)$ is a fundamental solution of polyharmonic equations.

Due to the identity

$$
\Delta[-\ln |x|]=\frac{2-n}{|x|^{2}}
$$

we have

Proposition 3.2. In the case where $n=2 m$,

$$
\Lambda_{n \mid \frac{n}{2}}(x)=-c_{n} \ln |x|
$$

is a fundamental solution of equation (1.1), where the constant $c_{n}$ depends only on the space dimension.

Corollary 3.1. The fundamental solution $\Lambda_{n \mid m}(x)$ can be represented by the fundamental solution of Laplace equation as

$$
\Lambda_{n \mid m}(x)=\frac{|x|^{2(m-1)}}{2^{(m-1)}(m-1) !} \frac{1}{\prod_{k=1}^{m}(2 k-n)} \Lambda_{n \mid 1}(x)
$$

when:

a) the space dimension is an arbitrary odd number;

b) the space dimension is an even number, but it is greater than the order of the equation (i.e., $n>2 m$ ).

Theorem 3.2. A generalized solution of the BVP (3.1) for equation (2.1) in the half-space $\Re_{+}^{n}$ is represented as

$$
u(x)=\int_{\Re_{+}^{n}} G_{n \mid m}(x, y) f(y) d y,
$$

where the Green's function has the form

$$
G_{n \mid m}(x, y)=c_{n m}|x-y|^{2} G_{n \mid m-1}(x, y)-\frac{\kappa_{n m} x_{n}^{m-1} y_{n}^{m-1}}{|x-\bar{y}|^{n-2}} ;
$$


here $k$ and $c_{n m}$ are constants, the latter being defined by (3.8) and Proposition 3.2 for $n=2 m$. This solution satisfies condition (3.2) and therefore it is unique.

Proof. By Almanzi's representation (2.13) the first term on the right-hand side of (3.18) is a fundamental solution. Also, by the representation (2.15) the second term of (3.18) is the polyharmonic function

$$
w_{1}(x)=\frac{\kappa_{n m} y_{n}^{m-1}}{|x-\bar{y}|^{n-2}} .
$$

Note that $\frac{\partial}{\partial \vec{\nu}} \equiv-\frac{\partial}{\partial x_{n}}$. So, for every $j=\overline{0, m-2}$, the partial derivatives of order $j<m-1$ of both terms on the right-hand side of (3.18) equal zero on the boundary. The constant $\kappa_{n m}$ is chosen so that a partial derivative of the function (3.18) of order $m-1$ vanishes for $x_{n}=0$. Therefore the solution (3.17) satisfies the boundary conditions (3.1). By the construction of the Green's function (3.18), it is easy to check that the condition (3.2) takes place when the space dimension is greater than $2 m$, the order of equation (1.1), and therefore this solution is unique.

To construct the Green's function and to simplify the calculation of higher order derivatives, let as use the following special notation: if $y \equiv\left(y_{1}, \ldots, y_{n}\right)$ is a point in $\Re_{l}^{n}$ and $\varsigma \equiv\left(\varsigma_{1}, \ldots, \varsigma_{n}\right)$ is a multiindex, where $\varsigma_{k}$ equals 1 or 0 for every $k=\overline{1, n}$, then

$$
y^{\varsigma} \equiv\left((-1)^{\varsigma_{1}} y_{1}, \ldots,(-1)^{\varsigma_{n}} y_{n}\right)
$$

Using this notation, we have

$$
\left|x-y^{\varsigma}\right| \equiv\left[\sum_{k=1}^{n}\left(x-(-1)^{\varsigma_{k}} y_{k}\right)^{2}\right]^{\frac{1}{2}}
$$

and it is easy to check that

$$
\frac{\partial\left|x-y^{\varsigma}\right|^{-k}}{\partial x_{j}}=\frac{-k\left[x_{j}-(-1)^{\varsigma_{j}} y_{j}\right]}{\left|x-y^{\varsigma}\right|^{k+2}}
$$

and

$$
\frac{\partial\left|x-y^{\varsigma}\right|^{-k}}{\partial y_{j}}=\frac{(-1)^{\varsigma_{j}} k\left[x_{j}-(-1)^{\varsigma_{j}} y_{j}\right]}{\left|x-y^{\varsigma}\right|^{k+2}} .
$$

Theorem 3.3. If $u(x)$ defined by (3.17) is a solution of the BVP (1.1), (3.1), then

$$
u(x)=c_{n m}\left[\int_{\Re_{+}^{n}} y_{n}^{m} f(y) d y\right] \cdot \frac{x_{n}^{m}}{|x|^{n}}+O\left(|x|^{m-n+1}\right) .
$$

Proof. Note that the coefficients of the first terms of the Taylor's series in a neighborhood of $y=0$ of the solution (3.17) are

$$
G_{n \mid m}(x, 0)=\left.\frac{\partial G_{n \mid m}(x, y)}{\partial y_{k}}\right|_{y=0}=\left.D^{\alpha} G_{n \mid m}(x, y)\right|_{y=0}=0
$$


for every $k=\overline{1, n}$ and $|\alpha| \leq m$, where $\alpha_{n} \neq m$. But

$$
\left.\frac{\partial^{m} G_{n \mid m}(x, y)}{\partial y_{n}^{m}}\right|_{y=0}=M_{n \mid m} \cdot \frac{x_{n}^{m}}{|x|^{n}} .
$$

\section{ACKNOWLEDGEMENT}

The author is deeply grateful to Prof. A. Koshelev and Prof. O. Chkadua for a useful discussion of the problems considered in this paper.

\section{REFERENCES}

1. A. V. Bitsadze, Some classes of partial differential equations. (Russian) Nauka, Moscow, 1981.

2. C. Cassisa, P. E. Ricci, and I. Tavkhelidze, Analogue of Saint-Venant's principle for the one special type 4 -th order elliptic equation and its applications. Appl. Math. Inform. 4(1999), No. 2, 11-29, 96.

3. O. Chkadua and R. Duduchava, Asymptotics of functions represented by potentials. Russ. J. Math. Phys. 7(2000), No. 1, 15-47.

4. D. G. Duffy, Green's functions with applications. Studies in Advanced Mathematics. Chapman \& Hall/CRC, Boca Raton, FL, 2001.

5. K. O. Friedrichs, On the boundary-value problems of the theory of elasticity and Korn's inequality. Ann. of Math. (2) 48(1947), 441-471.

6. A. M. Gomilko, The classical Dirichlet problem for the biharmonic equation in a halfstrip. (Russian) Differ. Uravn. 34(1998), No. 2, 228-237; English transl.: Differential Equations 34(1998), No. 2, 231-241.

7. L. HöRmander, Linear partial differential operators. Die Grundlehren der mathematischen Wissenschaften, Bd. 116. Academic Press, Inc., Publishers, New York; SpringerVerlag, Berlin-Göttingen-Heidelberg, 1963.

8. V. A. Kondrat'Ev Boundary value problems for elliptic equations in domains with conical or angular points. (Russian) Trudy Moskov. Mat. Obšč. 16(1967), 209-292.

9. V. A. Kondrat'ev, I. KopacheK, D. M. Lekveishvili, and O. A. Olě̌nik, Sharp estimates in Hölder spaces and the exact Saint-Venant principle for solutions of the biharmonic equation. (Russian) Modern problems of mathematics. Differential equations, mathematical analysis and their applications. Trudy Mat. Inst. Steklov. 166(1984), 91106.

10. V. A. Kondrat'Ev and O. A. Olěrnik, Boundary value problems for a system in elasticity theory in unbounded domains. Korn inequalities. (Russian) Uspekhi Mat. Nauk 43(1988), No. 5(263), 55-98; English transl.: Russian Math. Surveys 43(1988), No. 5, $65-119$

11. A. I. Koshelev, On the regularity up to the boundary of stresses in the linear theory of elasticity. (Russian) Dokl. Akad. Nauk 386(2002), No. 2, 194-197; English transl.: Dokl. Phys. 47(2002), No. 9, 690-692

12. V. Kozlov and V. Maz'Ya, Asymptotics of a singular solution to the Dirichlet problem for an elliptic equation with discontinuous coefficients near the boundary. Function spaces, differential operators and nonlinear analysis (Teistungen, 2001), 75-115, Birkhäuser, Basel, 2003.

13. P. D. LAX, A Phragmén-Lindelof theorem in harmonic analysis and its application to some questions in the theory of elliptic equations. Comm. Pure Appl. Math. 10(1957), $361-389$. 
14. O. A. Olĕ̌nIK, Lectures on partial differential equations. Part I. (Russian) Moskov. Gos. Univ., Mekh.-Mat. Fak., Moscow, 1976.

15. O. A. OleǏnik, G. A. Iosif'yan, and I. N. TAVkhelidze, Asymptotic behavior of the solution of a biharmonic equation in the neighborhood of nonregular points of the boundary of the domain and at infinity. (Russian) Trudy Moskov. Mat. Obshch. 42(1981), 166-175.

16. L. Schwartz, Theorie des distributions. Tome I. Actualites Sci. Ind., no. $1091=$ Publ. Inst. Math. Univ. Strasbourg 9. Hermann \& Cie., Paris, 1950; Tome II. Actualites Sci. Ind., no. 1122 = Publ. Inst. Math. Univ. Strasbourg 10. Hermann \& Cie., Paris, 1951.

17. S. L. Sobolev, On a boundary value problem for polyharmonic equations. (Russian) Mat. Sb., N. Ser. 2 (44)(1937), 467-499; English transl.: Amer. Math. Soc Transl. (2) 33(1963), 1-40.

18. S. L. Sobolev, Some applications of functional analysis in mathematical physics. (Russian) Izdat. Leningrad. Gos. Univ., Leningrad, 1950; English transl.: Translations of Mathematical Monographs, 90. American Mathematical Society, Providence, RI, 1991.

19. S. L. Sobolev, Cubature formulas and modern analysis. An introduction. (Translated from the 1988 Russian edition) Gordon and Breach Science Publishers, Montreux, 1992.

20. I. N. TAVkHELIDze, Asymptotic of solution of Dirichlet boundary value problem for polyharmonic equation in the polyhedral "canonical" angles. Semin. I. Vekua Inst. Appl. Math. Rep. 12(1997), No. 1, 30-31.

21. I. N. TAVkhelidze, On the behavior of solutions of the one special type 4-th order elliptic equation at the infinity. Semin. I. Vekua Inst. Appl. Math. Rep. 24(1998), 31-45.

22. A. N. Tikhonov and A. A. SAmarskit, Equations of mathematical physics. Nauka, Moscow, 1977.

23. I. N. VEKUA, On metaharmonic functions. Trudy Tbiliss. Mat. Inst. Razmadze 12(1943), 105-174.

24. I. N. VEKUA, New methods for solving elliptic equations. (Russian) OGIZ, MoscowLeningrad, 1948; English transl.: North-Holland Series in Applied Mathematics and Mechanics, Vol. 1. North-Holland Publishing Co., Amsterdam; Interscience Publishers John Wiley \& Sons, Inc., New York, 1967.

(Received 1.06.2007)

Author's address:

I. Vekua Institute of Applied Mathematics

I. Javakhishvili Tbilisi State University

2, University St., 0143 Tbilisi

Georgia

E-mail: i.tavkhelidze@math.sci.tsu.ge 